\title{
Synthesis, antimicrobial evaluation and docking studies of new pyrazolone derivatives
}

\author{
Abdulsalam AM Alkhaldi ${ }^{1}$, Mohamed A Abdelgawad ${ }^{2,3 *}$, Bahaa GM Youssif ${ }^{2,4}$ \\ ${ }^{1}$ Department of Biology, College of Science, Jouf University, Sakaka, Aljouf 2014, Saudi Arabia, ${ }^{2}$ Department of Pharmaceutical \\ Chemistry, College of Pharmacy, Jouf University, Sakaka, Aljouf 2014, Saudi Arabia, ${ }^{3}$ Department of Pharmaceutical Organic \\ Chemistry, Faculty of Pharmacy, Beni-Suef University, Beni-Suef $62514,{ }^{4}$ Department of Pharmaceutical Organic Chemistry, \\ Faculty of Pharmacy, Assiut University, Assiut 71526 Egypt
}

*For correspondence: Email: mohamedabdelwahab976@yahoo.com; Tel: 00202 38482600, +966-595435214

\begin{abstract}
Purpose: To synthesize new antimicrobial azo-pyrazolone derivatives III \& IV and evaluate their antimicrobial activities using a combination of in vitro and molecular docking studies.

Methods: Azopyrazolone compounds were prepared from the reaction of substituted aniline diazonium with ethyl acetoacetate to give azoxobutyric acid derivatives (II) which were then reacted with phenyl hydrazine or hydrazine hydrate. The pyrazolone derivatives (IV) were acetylated with glacial acetic acid to yield new acetylated pyrazolones (V). An agar dilution method was used to demonstrate the antimicrobial activities of the pyrazolone derivatives and their minimum inhibitory concentration (MIC) values calculated. Molecular docking studies were employed to further evaluate the most active compounds (on the basis of the MICs obtained).

Results: The new pyrazolone derivatives showed varying antimicrobial activities (from negligible to strong) against a number of microorganisms. Derivatives IIIb and Vb showed potent activities against Bacillus subtilis, Sarcina lutea, Staphylococcus aureus and Enterococcus faecalis. However, the new compounds did not show antifungal activity. Molecular docking results for compounds IIIb and Vb were consistent with their antimicrobial activities and proved that the compounds inhibited glucosamine-6phosphate synthase.

Conclusion: The new dichloropyrazolone compounds IIla and Vb possess potent antimicrobial activities. These compounds have promising potential for use as new antibacterial agents or as templates for the design of new antimicrobial drugs.
\end{abstract}

Keywords: Azo-pyrazolone, Dichloropyrazolone, Antimicrobial, Molecular docking

\begin{abstract}
This is an Open Access article that uses a funding model which does not charge readers or their institutions for access and distributed under the terms of the Creative Commons Attribution License (http://creativecommons.org/licenses/by/4.0) and the Budapest Open Access Initiative (http://www.budapestopenaccessinitiative.org/read), which permit unrestricted use, distribution, and reproduction in any medium, provided the original work is properly credited.

Tropical Journal of Pharmaceutical Research is indexed by Science Citation Index (SciSearch), Scopus, International Pharmaceutical Abstract, Chemical Abstracts, Embase, Index Copernicus, EBSCO, African Index Medicus, JournalSeek, Journal Citation Reports/Science Edition, Directory of Open Access Journals (DOAJ), African Journal Online, Bioline International, Open-J-Gate and Pharmacy Abstracts
\end{abstract}

\section{INTRODUCTION}

Literature survey has revealed that pyrazolone compounds exhibit a variety of biological activities [1-5]. For example, the azo-pyrazolone derivatives have potent cytotoxic [6,7], anti- inflammatory [8-10] and antibacterial activities [11]. Interestingly, pyrazolones $\mathbf{1}$ and 2 have antimicrobial activities and are considered novel types of non-nucleoside reverse transcriptase inhibitors against HIV-1 [12]. However, drazoxolon $\mathbf{3}$ is an azo compound containing 
isoxazolone ring (ring isostere of pyrazoles) attached to phenyl hydrazone, and is used as a commercial fungicidal agent [13]. In the review of reported biological activities of these derivatives, it was seen that a number of substituted phenylhydrazones bearing a pyrazole ring (IIla-c, IVa-c and Va-c) have been synthesized (Figure 1).
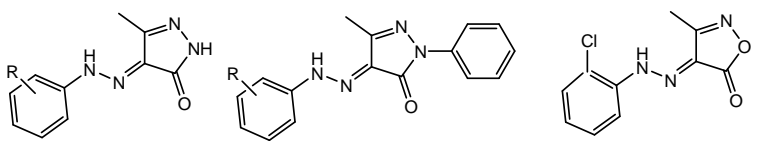<smiles>CC1=NNC(=O)C1=NNc1ccc(Br)cc1</smiles><smiles>CC(=O)N1N=C(C)C(N=C2CC3C=CC2C3)C1=O</smiles>

Figure 1: Structures of (a) pyrazolones compounds 1 - 3 and (b) new compounds containing pyrazolones (IIIa-c, IV a-c and Va-c)

The synthesis of new pyrazolone compounds may be a valuable way for producing novel antimicrobial drugs.

\section{EXPERIMENTAL}

\section{Instrumentation}

Melting points, NMR, IR, mass spectroscopy and elemental composition were measured in accordance with standard procedures reported in the literature [6-9]. All reagents used in this study were purchased from Aldrich Chemical Company (Milwaukee, WI). The authors synthesized compounds II b and IIc, and compounds IVb and Ilc in line with reported methods [14-16].

Synthesis of 2-[(3, 4-dimethoxy-phenyl)hydrazono]-3-oxo-butyric acid ethyl ester (II a)

To a mixture 3,4-dimethoxyaniline $(0.01 \mathrm{~mol})$ in aqueous $\mathrm{HCl}(10 \%, 10 \mathrm{~mL})$, an equal volume of ice-cooled $\mathrm{NaNO}_{2}$ solution was added in aliquots for over $20 \mathrm{~min}$ with vigorous stirring to afford the diazonium salt. To an ice-cooled solution of the ethyl acetoacetate $(0.01 \mathrm{~mol})$ and sodium acetate $(0.02 \mathrm{~mol})$ in aqueous ethanol $(50 \%, 20$ $\mathrm{mL}$ ), the diazonium salt was added for $3 \mathrm{~h}$ with stirring. Following filtration, the product was rinsed in water and purified by crystallization from methanol to yield compound Ila.

\section{Method used for synthesizing IIla-c}

A mixture of Ila-c $(0.015 \mathrm{~mol})$ in absolute ethanol $(20 \mathrm{~mL})$, and phenyl hydrazine $(0.03 \mathrm{~mol})$ was continually refluxed under heat for $8 \mathrm{~h}$. On cooling, the product was rinsed in water, and after drying, it was subjected to crystallization from acetic acid.

Synthesis of 4-[(3,4-dimethoxy-phenyl)hydrazono]-5-methyl-2,4-dihydropyrazo-3-one (IV a)

To a solution of II a (0.03) in absolute ethanol (20 $\mathrm{mL})$, hydrazine monohydrate $(0.03 \mathrm{~mol})$ was added and heated under reflux for $6 \mathrm{~h}$. The reaction was cooled and poured into crushed ice. The precipitate was filtered, washed with water and crystallized from ethanol.

\section{General procedure for synthesis of Va-c}

Pyrazolone compounds IV a-c $(0.01 \mathrm{~mol})$ were heated under reflux in glacial acetic acid $(30 \mathrm{~mL})$ for $8 \mathrm{~h}$. The reaction mixture was cooled to room temperature and added to ice-cooled water (100 $\mathrm{mL}$ ). The resultant precipitate was filtered, dried and crystallized from ethanol.

\section{Antimicrobial screening}

Minimum inhibitory concentration (MIC) was determined using agar dilution according to the procedures outlined by CLSI [17-20].

\section{Molecular modeling studies}

Docking experiments were performed using MOE ver. 2010.08. Crystalline structures of glucosamine-6-phosphate (Glcm6P) complexed with glucosamine-6-phosphate synthase (PDB: ID 2VF5) was provided by Protein Data Bank (RCSB). The Docking studies were carried out according to reported methods [6-9].

\section{RESULTS}

\section{Spectral data}

\section{2-[(3, 4-Dimethoxy-phenyl)-hydrazono]-3-oxo- butyric acid ethyl ester (II a)}

Red crystal, yield (85\%); mp $160-162^{\circ} \mathrm{C}$; IR (film) $3375(\mathrm{NH}), \quad 2974,2931,2912(\mathrm{CH}$, aliphatic), 1705 (broad band, $\mathrm{C}=\mathrm{O}) \mathrm{cm}^{-1} ;{ }^{1} \mathrm{H}$ NMR (DMSO- $\left.d_{6}\right) \delta$ 1.26-1.32(m, $\left.3 \mathrm{H}, \mathrm{CH}_{2} \mathrm{CH}_{3}\right)$, 2.37( $\left.\mathrm{s}, 3 \mathrm{H}, \mathrm{COCH}_{3}\right), 3.74\left(\mathrm{~s}, 3 \mathrm{H}, 3-\mathrm{OCH}_{3}\right)$, $3.79\left(\mathrm{~s}, 3 \mathrm{H} .4 \mathrm{OCH}_{3}\right), 4.20-4.30\left(\mathrm{~m}, 2 \mathrm{H},-\mathrm{CH}_{2} \mathrm{CH}_{3}\right)$ 6.95-7.07 ( $\mathrm{m}, 2 \mathrm{H}$, phenyl $\mathrm{H}-5,6)$ 7.13-7.22(m, $1 \mathrm{H}$, phenyl $\mathrm{H}-2), 11.80(\mathrm{~s}, 1 \mathrm{H}, \mathrm{NH}) ;{ }^{13} \mathrm{C} \mathrm{NMR}$ $\left(\mathrm{DMSO}^{-} d_{6}\right) \delta$ 14.39, 26.14, 55.88, 60.66, 61.29, 100.73, 107.39, 112.81, 125.14, 135.87, 146.23, 149.97, 163.25, 193.68; analytically calculated values for $\mathrm{C}_{14} \mathrm{H}_{16} \mathrm{~N}_{4} \mathrm{O}_{4}: \mathrm{C}, 55.26 ; \mathrm{H}, 5.30 ; \mathrm{N}$, 
18.41; obtained values: $\mathrm{C}, 55.50 ; \mathrm{H}, 5.20 ; \mathrm{N}$, 18.50 .

\section{4-[(3, 4-Dimethoxy-phenyl)-hydrazono]-5- methyl-2-phenyl-2,4-dihydropyrazol-3-one (III a)}

Dark red crystal, yield (60\%); $\mathrm{mp} 240-242{ }^{\circ} \mathrm{C}$; IR (film) $3425(\mathrm{NH}), 2900(\mathrm{CH}$, aliphatic ), 1651 (broad band, $\mathrm{C}=\mathrm{O}$ ) $\mathrm{cm}^{-1}$; ${ }^{1} \mathrm{H}$ NMR (DMSO- $d_{6}$ ) $\delta$ $2.30\left(\mathrm{~s}, 3 \mathrm{H}, \mathrm{CH}_{3}\right), \quad, 3.78\left(\mathrm{~s}, 3 \mathrm{H}, 3-\mathrm{OCH}_{3}\right)$, $3.82\left(\mathrm{~s}, 3 \mathrm{H} .4 \mathrm{OCH}_{3}\right), 7.03(\mathrm{~d}, \mathrm{~J}=8.8 \mathrm{~Hz}, 1 \mathrm{H}$, dimethoxyphenyl $\mathrm{H}-2) \quad 7.14-7.22(\mathrm{~m}, \quad 2 \mathrm{H}$, dimethoxyphenyl $\mathrm{H}-5,6), \quad 7.29-7.31(\mathrm{~m}, 1 \mathrm{H}$, phenyl $\mathrm{H}-4), 7.43-7.47(\mathrm{~m}, 2 \mathrm{H}$, phenyl $\mathrm{H}-3,5)$, 7.93( $\mathrm{d}, \mathrm{J}=8 \mathrm{~Hz}, 2 \mathrm{H}$, phenyl H-2,5), $13.38(\mathrm{~s}, 1 \mathrm{H}$, $\mathrm{NH}) ;{ }^{13} \mathrm{C}$ NMR (DMSO- $\left.d_{6}\right) \delta 12.08,56.10,56.27$, $101.23,108.87,112.81,118.08,125.14,127.03$, 129.46, 135.44, 138.56, 147.76, 148.70, 150.08, 157.24; analytically calculated values for $\mathrm{C}_{18} \mathrm{H}_{18} \mathrm{~N}_{4} \mathrm{O}_{3}$ : C, 63.89; $\mathrm{H}, \quad 5.36 ; \mathrm{N}$, 16.56; obtained values: $\mathrm{C}, 63.80 ; \mathrm{H}, 5.30 ; \mathrm{N}, 16.60$.

\section{4-[(3, 5-Dichloro-phenyl)-hydrazono]-5- methyl-2-phenyl-2,4-dihydro-pyrazol-3-one (III b)}

Pale red crystal, yield (55\%); mp $282-284{ }^{\circ} \mathrm{C}$; IR (film) 3396,3232 (NH), 3089(CH, aromatic), 2900 ( $\mathrm{CH}$, aliphatic), $1654 \quad(\mathrm{C}=\mathrm{O}) \mathrm{cm}^{-1} ;{ }^{1} \mathrm{H}$ NMR (DMSO-d $\left.d_{6}\right) \delta 2.30\left(\mathrm{~s}, 3 \mathrm{H}, \mathrm{CH}_{3}\right), 7.22-7.24(\mathrm{~m}, 1 \mathrm{H}$, phenyl $\mathrm{H}-4), 7.38(\mathrm{~s}, 1 \mathrm{H}$, dichlorophenyl $\mathrm{H}-4)$, 7.44-7.46(m, 2H, Phenyl H-3, 5), 7.70- (s, 2H, dichlorophenyl $\mathrm{H}-2,6)$, 7.89(d, $J=8 \mathrm{~Hz}, 2 \mathrm{H}$, phenyl $\mathrm{H}-2,6)$; Mass spectra, $\mathrm{M}^{+}=346,100 \%$, $[\mathrm{M} .+2]^{+}=348,64 \%$, analytically calculated values for $\mathrm{C}_{16} \mathrm{H}_{12} \mathrm{Cl}_{2} \mathrm{~N}_{4} \mathrm{O}$ : C, 55.35; $\mathrm{H}, 3.48 ; \mathrm{N}$, 16.14; obtained values: $\mathrm{C}, 55.30 ; \mathrm{H}, 3.20 ; \mathrm{N}$, 16.30 .

\section{4-[N-(3-methyl--5-oxo-1-phenyl-1,5- dihydropyrazo-4-ylidine)-hydrazino]-benzoic acid ethyl ester (IIIc)}

Pale yellow crystal, yield $50 \%$; mp $250-252^{\circ} \mathrm{C}$; IR (film) $3313(\mathrm{NH}), 3055(\mathrm{CH}$, aromatic ), 2974, $2960\left(\mathrm{CH}\right.$, aliphatic), 1720, $1670(2 \mathrm{C}=0) \mathrm{cm}^{-1}$; ${ }^{1} \mathrm{H}$ NMR (DMSO- $\left.d_{6}\right) \delta 1.29(\mathrm{t}, J=6.8,3 \mathrm{H}$, $\left.\mathrm{CH}_{2} \mathrm{CH}_{3}\right), 2.23\left(\mathrm{~s}, 3 \mathrm{H}, \mathrm{CH}_{3}\right), 4.26(\mathrm{q}, \mathrm{J}=6.8$, $\left.2 \mathrm{H},-\mathrm{CH}_{2} \mathrm{CH}_{3}\right), 7.20-7.23(\mathrm{~m}, 1 \mathrm{H}$, phenyl $\mathrm{H}-4)$, 7.43-7.46( $\mathrm{m}, 2 \mathrm{H}$, disubstituted-phenyl $\mathrm{H}-3,5)$, 7.60-7.63(m, 2H, phenyl H-3, 5), $7.88(\mathrm{~d}, 2 \mathrm{H}, \mathrm{J}=$ 7.6 Hz, disubstituted-phenyl $\mathrm{H}-2, \mathrm{H}-6)$, 7.937.97(m, 2H, phenyl $\mathrm{H}-2,6), 13.10(\mathrm{~s}, 1 \mathrm{H}, \mathrm{NH})$; ${ }^{13} \mathrm{C}$ NMR (DMSO- $\left.d_{6}\right) \delta 12.15,14.66,61.15$, $116.40,118.20,125.43,126.61,129.53,131.26$, $137.20, \quad 138.25,145.25, \quad 147.40, \quad 149.21$, 165.60.; analytically calculated values for
$\mathrm{C}_{19} \mathrm{H}_{18} \mathrm{~N}_{4} \mathrm{O}_{3}: \quad \mathrm{C}, \quad 65.13 ; \mathrm{H}, \quad 5.18 ; \mathrm{N}, \quad 15.99 ;$ obtained values: C, 65.20; H, 5.00; N, 15.70 .

\section{Synthesis of 4-[(3, 4-dimethoxy-phenyl)- hydrazono]-5-methyl-2,4-dihydropyrazo-3-one (IVa)}

A mixture of Ila (0.03) in absolute ethanol (20 $\mathrm{mL})$ and hydrazine monohydrate $(0.03 \mathrm{~mol})$ was refluxed with heating for $6 \mathrm{~h}$. The reaction mixture was cooled and the product was obtained by precipitating in ice. It was thereafter sieved, washed with water and subjected to crystallization in ethanol. Red crystal, yield (80 $\%$ ); mp 232-234C; IR (film) 3329 (NH), 3020( $\mathrm{CH}$, aromatic), 2966, 2927 ( $\mathrm{CH}$ aliphatic), 1658 (broad band, $\mathrm{C}=\mathrm{O}) \mathrm{cm}^{-1}$; ${ }^{1} \mathrm{H}$ NMR (DMSO- $\left.d_{6}\right) \delta$ $2.15\left(\mathrm{~s}, 3 \mathrm{H}, \mathrm{CH}_{3}\right), 3.76\left(\mathrm{~s}, 3 \mathrm{H}, 3-\mathrm{OCH}_{3}\right), 3.80$ $\left(\mathrm{s}, 3 \mathrm{H} .4 \mathrm{OCH}_{3}\right), \quad 6.99(\mathrm{~d}, 1 \mathrm{H}, \mathrm{J}=8.8 \mathrm{~Hz}$, phenyl $\mathrm{H}-5$ ), 7.04 (dd, $J_{1}=8.8 \mathrm{H}_{z}, J_{2}=2 \mathrm{~Hz}, 1 \mathrm{H}$, phenyl $\mathrm{H}-6), 7.19$ ( $\mathrm{s}, 1 \mathrm{H}, \mathrm{J}=2 \mathrm{~Hz}$, phenyl $\mathrm{H}-2), 11.48$ (s, $1 \mathrm{H}, \quad \mathrm{NH}) ;{ }^{13} \mathrm{C}$ NMR (DMSO- $\left.d_{6}\right) \delta, 12.05$, 56.06, 56.25, 100.80, 108.09, 112.90, 127.44, $135.63, \quad 147.01, \quad 147.22, \quad 150.10, \quad 160.79$; analytically calculated values for $\mathrm{C}_{12} \mathrm{H}_{14} \mathrm{~N}_{4} \mathrm{O}_{3}$ : C, 54.96; $\mathrm{H}, 5.38 ; \mathrm{N}, 21.36$; obtained values: $\mathrm{C}$, $54.80 ; \mathrm{H}, 5.50 ; \mathrm{N}, 21.40$.

\section{2-Acetyl-4-[(3,4-dimethoxy-phenyl)- hydrazono]-5-methyl-2,4-dihydropyrazo-3-one (Va)}

Yellowish red crystal, yield (70 \%); mp 292-294 ${ }^{\circ} \mathrm{C}$; IR (film) $3329(\mathrm{NH}), 2962$ (CH aliphatic), 1658 (broad band, $\mathrm{C}=\mathrm{O}$ ) $\mathrm{cm}^{-1}$; ${ }^{1} \mathrm{H}$ NMR (DMSO$\left.d_{6}\right) \delta 2.13\left(\mathrm{~s}, 3 \mathrm{H}, \mathrm{CH}_{3}\right), 2.21\left(\mathrm{~s}, 3 \mathrm{H}, \mathrm{COCH}_{3}\right)$, $3.77\left(\mathrm{~s}, 3 \mathrm{H}, 3-\mathrm{OCH}_{3}\right), 3.81\left(\mathrm{~s}, 3 \mathrm{H} .4-\mathrm{OCH}_{3}\right), 6.99$ ( $\mathrm{d}, 1 \mathrm{H}, J=8.8 \mathrm{~Hz}$, phenyl $\mathrm{H}-5), \quad 7.06(\mathrm{dd}$, $\mathrm{J}_{1}=8.8 \mathrm{H}_{\mathrm{z}}, \mathrm{J}_{2}=2 \mathrm{~Hz}, 1 \mathrm{H}$, phenyl $\left.\mathrm{H}-6\right), 7.20(\mathrm{~d}, 1 \mathrm{H}$, $J=2 \mathrm{~Hz}$, phenyl $\mathrm{H}-2), 11.49(\mathrm{~s}, 1 \mathrm{H}, \mathrm{NH}) ;{ }^{13} \mathrm{C}$ NMR (DMSO- $d_{6}$ ) $\delta$ 12.05, 56.07, 56.26, 100.82, 108.08, 112.92, 127.45, 135.63, 147.01, 147.22, 150.10, 160.79; Anal. Calcd for $\mathrm{C}_{14} \mathrm{H}_{16} \mathrm{~N}_{4} \mathrm{O}_{4}$ : C, 55.26; H, 5.30; N, 18.41. Found: C, 55.50; $\mathrm{H}$, $5.20 ; \mathrm{N}, 18.50$.

\section{2-Acetyl-4-[(3,5-dichloro-phenyl)-hydrazono]- 5-methyl-2,4-dihydro-pyrazol-3-one (Vb)}

Reddish yellow crystal, yield (60\%); mp 250-252 ${ }^{\circ} \mathrm{C}$; IR (film) $3251(\mathrm{NH}), 3062(\mathrm{CH}$, aromatic), $2900(\mathrm{CH}$, aliphatic $), 1739,1660(\mathrm{C}=\mathrm{O}) \mathrm{cm}^{-1}$; ${ }^{1} \mathrm{H}$ NMR (DMSO-d $)_{6}$ ) $\delta 1.91\left(\mathrm{~s}, 3 \mathrm{H}, \mathrm{CH}_{3}\right), 2.15($ $\mathrm{s}, 3 \mathrm{H}, \mathrm{COCH}_{3}$ ), 7.32(s, $1 \mathrm{H}$, phenyl H-4), 7.60 ( $\mathrm{s}$, $2 \mathrm{H}$, phenyl $\mathrm{H}-2,6), 11.61$ (s, $1 \mathrm{H}, \mathrm{NH}-\mathrm{N}=\mathrm{C}$ ).; Mass spectra, $[\mathrm{M}]^{+}=312,15.2 \%[\mathrm{M}+2]^{+}=348$, $64 \%$; analytically calculated values for $\mathrm{C}_{12} \mathrm{H}_{10} \mathrm{Cl}_{2}$ 
$\mathrm{N}_{4} \mathrm{O}_{2}$ : C, 46.03; $\mathrm{H}, 3.22 ; \mathrm{N}, 17.89 ;$ values obtained: C, 46.10; H, 3.10; N, 17.80.

\section{4-[N-(1-Acetyl-3-methyl-5-oxo-1,5- dihydropyrazo-4-ylidine)-hydrazino]-benzoic acid ethyl ester (Vc)}

Reddish yellow crystal, yield (55\%); mp 296-298 ${ }^{\circ} \mathrm{C}$; IR (film) 3294 (NH), 2978, $2927(\mathrm{CH}$, aliphatic), 1705, 1674 (2 $\mathrm{C}=\mathrm{O}) \mathrm{cm}^{-1} ;{ }^{1} \mathrm{H}$ NMR $\left(\mathrm{DMSO}-d_{6}\right) \delta 1.29\left(\mathrm{t}, \mathrm{J}=6.8,3 \mathrm{H},-\mathrm{CH}_{2} \mathrm{CH}_{3}\right), 1.90$ $\left(\mathrm{s}, 3 \mathrm{H}, \mathrm{CH}_{3}\right), 2.05\left(\mathrm{~s}, 3 \mathrm{H}, \mathrm{COCH}_{3}\right), 4.24(\mathrm{q}, \mathrm{J}=$ $\left.6.8,2 \mathrm{H},-\mathrm{CH}_{2} \mathrm{CH}_{3}\right), 7.46(\mathrm{~d}, 2 \mathrm{H}, \mathrm{J}=8.8$, phenyl $\mathrm{H}$ 3,5), 7.89 (d, 2H, phenyl H-2,6), 11.60 (s, 1H, $\mathrm{NH}) ;{ }^{13} \mathrm{C}$ NMR (DMSO- $\left.d_{6}\right) \delta 12.00,14.60,21.48$, 61.02, 115.59, 125.94, 130.28, 131.18, 131.47, 145.63, 147.35, 160.37, 165.55; analytically calculated values for $\mathrm{C}_{15} \mathrm{H}_{16} \mathrm{~N}_{4} \mathrm{O}_{4}: \mathrm{C}, 56.96 ; \mathrm{H}$, 5.10; N, 17.71; values obtained: C, 56.60; $\mathrm{H}$, $5.00 ; \mathrm{N}, 17.70$.

\section{Antimicrobial activity of synthesized compounds (MIC)}

The compounds showed good antibacterial activities against Pseudomonas aeruginosa especially samples IIlb and $\mathrm{Vb}$, which showed high activity against gram positive (Enterococcus faecalis, Staphylococcus aureus and Sarcina lutea) and gram negative (Salmonella typhi, E. coli and Pseudomonas aeruginosa) organisms, as well as Gram positive rods (Bacillus subtilis). All compounds were inactive against fungi (Candida albicans). Their MICs are shown in Table 1.

\section{DISCUSSION}

Compound Ila had the characteristic NMR peaks of methyl and ethyl groups. The cyclization of compounds Ila-c with phenyl hydrazine afforded the poly-substituted pyrazolone III. The HNMR of pyrazolones III a-c showed increasing number of aromatic proton peaks and disappearance of the ethyl group peaks. The butyric diazo compound II was reacted with hydrazine hydrate to give the new pyrazolone compounds IV a-c. Disappearance of aliphatic ethyl group HNMR peaks and appearance of $\mathrm{NH}$ peak confirmed the structure of compound IV a. The pyrazolone compounds IV a-c were acetylated with glacial acetic acid to produce the new acetylated pyrazolones $\mathrm{V}$ a-c. The appearance of new methyl of acetyl group peak and disappearance of $\mathrm{NH}$ peak confirmed the structure of compounds Va-c.

The dichlorophenyl group in pyrazolones IIIb and $\mathrm{Vb}$ has important role in the antibacterial activities of the pyrazolone compounds. In addition, the substitution at the nitrogen atom of pyrazolone with acetyl or phenyl ring enhances their activities.

To identify the possible mechanism of action of the newly synthesized compounds, they were subjected to molecular docking studies with glucosamine-6-phosphate synthase using the program of MOE site finder. D-glucosamine-6phosphate (D-Glcm6P) is produced from Dfructose-6-phosphate using L-glutamine as nitrogen source in a reaction catalyzed by glucosamine-6-phosphate synthase. Thus, the inhibition of glucosamine-6-phosphate synthase results in inhibition of production of $\mathrm{N}$ acetylglucosamine which is a major unit in bacterial cell wall building. Consequently, the enzyme is an essential target for antibacterial agents. The docking study was performed using reported methods [6-9]. In this study, the most active compounds IIIlb and $\mathbf{V b}$ from antimicrobial studies were docked into MurA-F binding site to confirm the ability of the novel candidates to act as antibacterial agents.

Table 1: MIC of samples against different microorganisms $(\mu \mathrm{g} / \mathrm{mL})$

\begin{tabular}{|c|c|c|c|c|c|c|c|c|}
\hline \multirow{2}{*}{$\begin{array}{l}\text { Synthesized } \\
\text { compound ( } 200-0.7 \\
\mu \mathrm{g} / \mathrm{mL})\end{array}$} & \multicolumn{3}{|c|}{ Gram -ve rods } & \multirow{2}{*}{$\begin{array}{c}\begin{array}{c}\text { Gram } \\
\text { +ve rods }\end{array} \\
\text { B. subtilis }\end{array}$} & \multicolumn{3}{|c|}{ Gram +ve cocci } & \multirow{2}{*}{$\begin{array}{c}\text { Fungi } \\
C . \\
\text { albicans }\end{array}$} \\
\hline & $\begin{array}{c}S . \\
\text { typhi }\end{array}$ & $\begin{array}{c}P . \\
\text { aeruginosa }\end{array}$ & $\begin{array}{c}E . \\
\text { coli }\end{array}$ & & $\begin{array}{c}\text { S. } \\
\text { Iutea }\end{array}$ & $\begin{array}{c}\text { S. } \\
\text { aureus }\end{array}$ & $\begin{array}{c}E . \\
\text { faecalis }\end{array}$ & \\
\hline III a & $>200$ & $>200$ & $>200$ & $>200$ & $>200$ & $>200$ & $>200$ & $>200$ \\
\hline Illb & $>200$ & $>200$ & $>200$ & $\leq 12.5$ & $\leq 12.5$ & $\leq 12.5$ & $\leq 12.5$ & $>200$ \\
\hline IIIC & $>200$ & $>200$ & $>200$ & 25 & $\leq 12.5$ & $>200$ & $>200$ & $>200$ \\
\hline Iva & $>200$ & $>200$ & $>200$ & $\leq 12.5$ & $\leq 12.5$ & $>200$ & $>200$ & $>200$ \\
\hline $\mathrm{IVb}$ & $>200$ & $\leq 12.5$ & $>200$ & $>200$ & $>200$ & $>200$ & $>200$ & $>200$ \\
\hline IV C & $>200$ & $>200$ & $>200$ & $\leq 12.5$ & $\leq 12.5$ & $\leq 12.5$ & $\leq 12.5$ & $>200$ \\
\hline $\mathrm{Va}$ & $>200$ & $>200$ & $>200$ & $>200$ & $>200$ & $>200$ & $>200$ & $>200$ \\
\hline $\mathrm{Vb}$ & $>200$ & $\leq 12.5$ & $>200$ & $\leq 12.5$ & $\leq 12.5$ & $\leq 12.5$ & $\leq 12.5$ & $>200$ \\
\hline Vc & $>200$ & $>200$ & $>200$ & $\leq 12.5$ & $\leq 12.5$ & $>200$ & $\leq 12.5$ & $>200$ \\
\hline Cefotaxime & 0.7 & 6.25 & 0.7 & 50 & 0.7 & 1.5 & $>200$ & $>200$ \\
\hline Ampicillin & 6.25 & $>200$ & 6.25 & 6.25 & 0.7 & 0.7 & 1.5 & $>200$ \\
\hline Fluconazole & $>200$ & $>200$ & $>200$ & $>200$ & $>200$ & $>200$ & $>200$ & 12.5 \\
\hline
\end{tabular}


Glucosamine-6-phosphate synthetase in complex with Glcm6P as a ligand was sourced from a data bank (PDB: ID 2VF5). The energy (S) of re-docking of Glcm6P into glucosamine-6phosphate synthase was- $15.39 \mathrm{kcal} / \mathrm{mol}$. Ala602, Val399, Thr302, Gln348, Ser303, Ser349, Ser347 and Thr352 contributed nine Hbonds involving $-\mathrm{NH}_{2},-\mathrm{OH}$ and $-\mathrm{PO}_{4}$ groups (Figure 2).
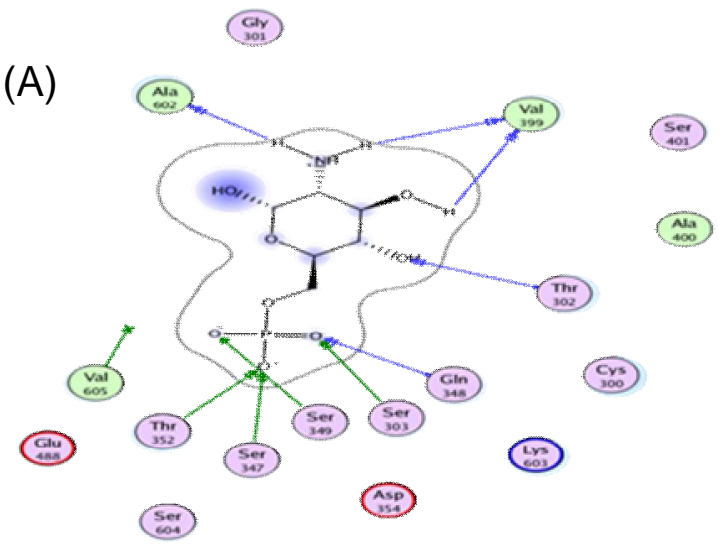

(B)

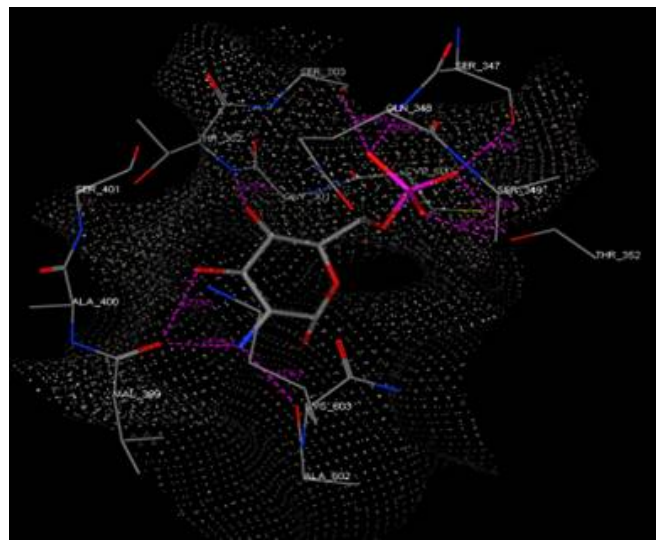

Figure 2: Glcm6P bound inside Glucosamine-6phosphate synthase. (A) 2D interactions of Glm6P within glucosamine-6-phosphate synthase $(\mathrm{H}$-bonds between $-\mathrm{NH}_{2},-\mathrm{OH}$ and $-\mathrm{PO}_{4}$ groups and Ala602, Val399, Thr302, Gln348, Ser303, Ser349, Ser347 and Thr352 amino acids are shown in red line dots. (B) 3D interactions of Glm6P

The results obtained in the docking studies are shown in Table 2. Compound IIIb exhibited better docking score $(-17.43 \mathrm{kcal} / \mathrm{mol})$ than Glm6P score energy $(S)=-15.39 \mathrm{kcal} / \mathrm{mol}$. Compound IIlb showed two hydrogen bonding interactions with Val399 and Ala602 (Figure 3).

Finally, the candidate $\mathbf{V b}$ showed an energy score of $-16.33 \mathrm{Kcal} / \mathrm{mol}$ and exhibited four hydrogen bonding interactions as follows: (i) Ala602 with $\mathrm{NH}$, (ii) Ser303 with $\mathrm{C}=\mathrm{O}$, (iii) Gln348 with $\mathrm{C}=\mathrm{O}$ and (iv) Thr352 with pyrazole $\mathrm{C}=\mathrm{O}$ (Figure- 4).
(A)

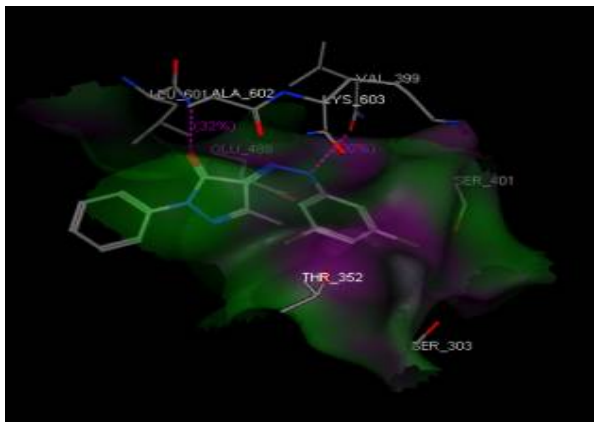

(B)

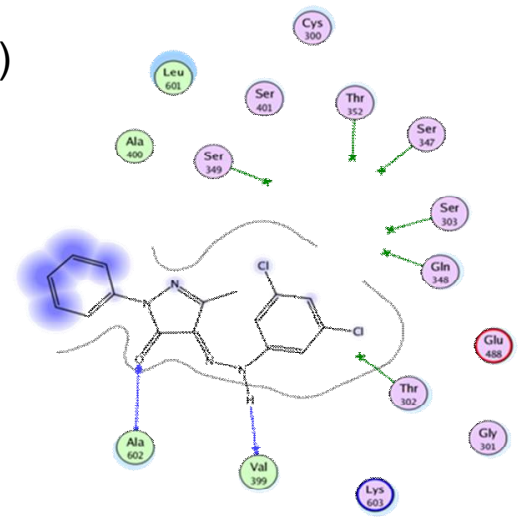

Figure 3: Binding of compound IIIb inside Glucosamine-6-phosphate synthase. (A) 2D interactions of Illb within Glucosamine-6-phosphate synthase, the dotted lines depict two $\mathrm{H}$-bonds between $\mathrm{NH}$ with Val399, and $\mathrm{C}=\mathrm{O}$ with Ala602. (B) 3D interactions of Vlb with Glucosamine-6-phosphat synthase

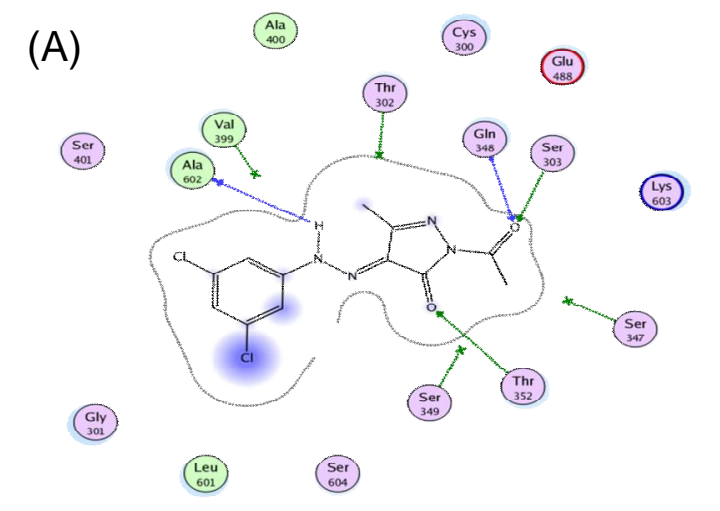

(B)

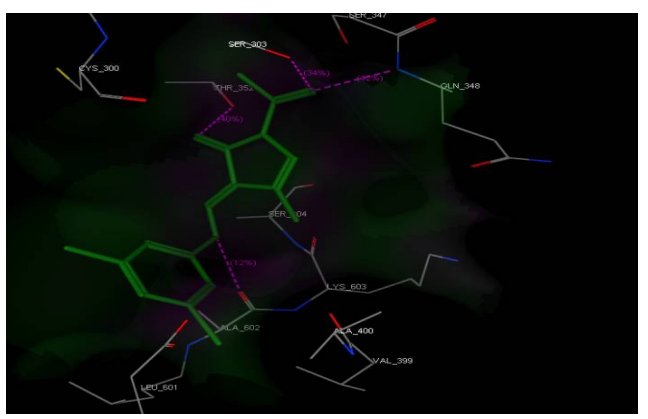

Figure 4: Binding of compound $\mathrm{Vb}$ inside glucosamine-6-phosphate synthase. (A) 2D interactions of $\mathrm{Vb}$ within Glucosamine-6-phosphate synthase (H-bonds are shown in red dots). (B) 3D interactions of VIII with GIm6P

Trop J Pharm Res, November 2018; 17(11): 2239 
Table 3: Docking results for IIIb, Vb and Glm6P

\begin{tabular}{|c|c|c|c|c|c|}
\hline $\begin{array}{l}\text { Synthesized } \\
\text { compound }\end{array}$ & $\begin{array}{l}\text { Affinity } \\
\text { (kcal/mol) }\end{array}$ & H-bonds (n) & $\begin{array}{l}\text { Space } \\
\text { residue }\end{array}$ & main & Group presen \\
\hline IIIb & -17.43 & 2 & $\begin{array}{l}\text { Val399 } \\
\text { Ala602 }\end{array}$ & $\begin{array}{l}3.24 \\
2.11\end{array}$ & $\begin{array}{l}\mathrm{NH} \\
\mathrm{C}=\mathrm{O}\end{array}$ \\
\hline Vb & -16.33 & 4 & $\begin{array}{l}\text { Ala602 } \\
\text { Ser303 } \\
\text { Gln348 } \\
\text { Thr352 }\end{array}$ & $\begin{array}{l}2.18 \\
3.15 \\
2.58 \\
2.48\end{array}$ & $\begin{array}{l}\mathrm{NH} \\
\mathrm{C}=\mathrm{O} \\
\mathrm{C}=\mathrm{O} \\
\text { Pyrazole } \mathrm{C}=\mathrm{O}\end{array}$ \\
\hline $\begin{array}{l}\text { Ligand } \\
\text { (Glucosamine - } \\
\text { 6-phosphate) }\end{array}$ & -15.39 & 9 & $\begin{array}{l}\text { Ala602 } \\
\text { Val399 } \\
\text { Thr302, } \\
\text { Gln348 } \\
\text { Ser303 } \\
\text { Ser349 } \\
\text { Ser347 } \\
\text { Thr352 }\end{array}$ & $\begin{array}{l}2.75 \\
2.46,2.52 \\
3.41 \\
2.11 \\
3.15 \\
3.20 \\
2.22 \\
3.21\end{array}$ & $\begin{array}{l}\mathrm{NH} \\
\mathrm{NH}, \mathrm{OH} \\
\mathrm{OH} \\
\mathrm{P}=\mathrm{O} \\
\mathrm{P}=\mathrm{O} \\
\mathrm{P}-\mathrm{O} \\
\mathrm{P}-\mathrm{O} \\
\mathrm{P}-\mathrm{O}\end{array}$ \\
\hline
\end{tabular}

\section{CONCLUSION}

The new pyrazolones, IIIa-c, IV a-c and V a-c, containing meta dichloro phenyl group, have promising antibacterial activity. The relationship between the docking results and their antibacterial activities provides important evidence for the mechanism of the new azopyrazolones as potent inhibitors of glucosamine-6-phosphate synthase. However, further studies are required to screen them for cytotoxic effects.

\section{DECLARATIONS}

\section{Acknowledgement}

The authors appreciate Jouf University, KSA, for funding the work through a research grant (Project no. 378/37). The authors would like to thank Dr Ahmed O El-Gendy and Dr Rania B Baker for helping with antimicrobial screening and molecular docking studies.

\section{Conflict of Interest}

No conflict of interest associated with this work.

\section{Contribution of Authors}

The authors declare that this work was done by the authors named in this article and all liabilities pertaining to claims relating to the content of this article will be borne by them.

\section{REFERENCES}

1. Bansal S, Bala $M$, Suthar SK, Choudhary $S$, Bhattacharya S, Bhardwaj $V$, Singla $S$, Joseph $A$. Design and synthesis of novel 2-phenyl-5-(1,3-diphenyl-
1H-pyrazol-4-yl)-1,3,4-oxadiazoles as selective COX-2 inhibitors with potent anti-inflammatory activity. Eur. J. Med. Chem. 2014; 80: 167-174.

2. Abd-El Gawad NM, Georgey HH, Ibrahim NA, Amin NH, Abdelsalam RM. Synthesis of novel pyrazole and dihydropyrazoles derivatives as potential antiinflammatory and analgesic agents. Arch. Pharm. Res. 2012; 35: 807-821.

3. Riyadh SM, Farghaly TA, Abdallah MA, Abdalla MM, Abd El-Aziz MR. New pyrazoles incorporating pyrazolylpyrazole moiety: Synthesis, anti-HCV and antitumor activity. Eur. J. Med. Chem. 2010; 45: 10421050.

4. Sayed HH, Hashem Al, Yousif NM, El-Sayed WA. Conversion of 3-Arylazo-5-phenyl-2(3H)-furanones into Other Heterocycles of Anticipated Biological Activity. Arch. Pharm. Chem 2007; 340; 315-319.

5. Kasımoğulları $R$, Bülbül $M$, Seçkin $B S$, Gökçe $B$. Synthesis, characterization and antiglaucoma activity of some novel pyrazole derivatives of 5-amino-1,3,4thiadiazole-2-sulfonamide. Eur. J. Med. Chem. 2010; 45: 4769-4773.

6. Abdelgawad MA, Bakr RB, Omar HA. Design, synthesis and biological evaluation of some novel benzothiazole/ benzoxazole and/ or benzimidazole derivatives incorporating a pyrazole scaffold as antiproliferative agents. Bioorganic Chem 2017; 74:82-9.

7. Belal A, Abdelgawad MA. New benzothiazole/ benzoxazole-pyrazole hybrids with potential as COX inhibitors: design, synthesis and anticancer activity evaluation. Res. Chem. Intermed 2017; 43(7): 38593872.

8. Abdelgawad MA, Labib MB, Abdel-Latif M. Pyrazolehydrazone derivatives as anti-inflammatory agents: Design, synthesis, biological evaluation, COX-1,2/5-LOX inhibition and docking study. Bioorganic Chem 2017; 74: 212-220.

9. Abdelgawad MA, Labib MB, Waleed AM, Kamel G, Azouz AA, EL-Shaymaa E. Design, synthesis, analgesic, anti-inflammatory activity of novel 
pyrazolones possessing aminosulfonyl pharmacophore as inhibitors of COX-2/5-LOX enzymes: Histopathological and docking studies. Bioorganic Chem 2018; 78: 103-114.

10. Abdelgawad MA, Bakr RB, Azouz AA. Novel pyrimidinepyridine hybrids: Synthesis, cyclooxygenase inhibition, anti-inflammatory activity and ulcerogenic liability; Bioorganic Chem 2018; 77: 339-348.

11. Oraby AK, Abdellatif KRA, Abdelgawad MA, Attia KA, Dawe LN, Georghiou PE. 2,4-Disubstituted Phenylhydrazonopyrazolone and Isoxazolone Derivatives as Antibacterial Agents: Synthesis, Preliminary Biological Evaluation and Docking Studies. Chem Select 2018; 3: 3295-3301.

12. Singh UP, Bhat HR, Verma A, Kumawat MK, Kaur R, Gupta SK, Singh RK. Phenyl hydrazone bearing pyrazole and pyrimidine scaffolds: design and discovery of a novel class of non-nucleoside reverse transcriptase inhibitors (NNRTIs) against HIV-1 and their antibacterial properties. RSC Advances 2013; 3(38): 17335-17348.

13. Vyas DH, Tala SD, Dhaduk MF, Akbari JD, Joshi HS. Synthesis, antitubercular and antimicrobial activities of some new pyrazoline and isoxazole derivatives. J. Indian Chem Soc 2007; 84: 1140-1144.

14. Kalluraya, Balakrishna K, Arun I, Priya FV, Jagadeesha $R L$. Synthesis and pharmacological activity of 4(substituted)-2-[4-aryl-hydrazono-3-methyl-5-oxo-2pyrazolin-1-yl]-thiazoles. Indian J. Heterocycl. Chem. 2004; 13(3): 245-248.
15. Etman HA, Sadek MG, Khalil AM. Synthesis of Some New Heterocycles Derived from 3-Amino5hydrazinopyrazole dihydrochloride. Res. J. Pharm., Biol. Chem. Sci. 2015; 6(2): 247-254.

16. Bakulev VA, Berseneva VS, Belskaia NP, Morzherin YY, Zaitsev A, Dehaen W, Ingrid L, Toppe S. Reactions of 5mercaptoazoles and pyridine-2-thiones with acetylenic esters. Selectivity of the formation of novel fused thiazin4-ones and thiazolidin-4-ones. Org. Biomol. Chem. 2003; 1: 134-139.

17. Sader HS, Flamm RK, Jones RN. Antimicrobial activity of daptomycin tested against Gram-positive pathogens collected in Europe, Latin America, and selected countries in the Asia-Pacific Region. Diagn Microbiol Infect Dis 2013; 75: 417-422.

18. Tarek N, Hassan HM, AbdelGhani SM, Radwan IA, Hammouda OE, Gendy AO. Comparative chemical and antimicrobial study of nine essential oils obtained from medicinal plants growing in Egypt; Beni-Seuf Univ J Appl Sci 2014; 3(2): 149-156.

19. Hassana GS, Awdallaha MF, El-Saadib MT, Elgendic $A O$, Hemeda LR. Synthesis of some benzothiazole derivatives evaluated as antimicrobials and antibiofilms. J Chem Pharm Res 2015; 7(7):1125-1143.

20. Lamie PF, Philoppes JN, El-Gendy AO, Rarova L, Gruz J. Design, Synthesis and Evaluation of Novel Phthalimide Derivatives as in Vitro Anti-Microbial, AntiOxidant and Anti-Inflammatory Agents. Molecules 2015; 20(9): 16620-16642. 